\title{
Study on the Comparison of Chinese-English Synonyms
}

\author{
Qiuzhong OU \\ Wuhan Business University, Wuhan 430056, China
}

\begin{abstract}
In this thesis a comparison between Chinese and English synonyms has been made in terms of concept, classification, definition, differentiation standards, synonymous fields, and so on; and this thesis also points out the importance of comparison studies on Chinese and English synonyms. Through comparison, it is found that there is practically no difference between Chinese and English synonyms in concept, classification, and definition. There may be several differentiation measures or standards for word discrimination in a synonymous semantic field. There is little difference in macro distribution of differentiation standards between Chinese and English synonymous semantic fields but much, in micro distribution. It is up to the number of the semantic fields or clusters under a certain discriminative standard. Therefore, it is suggested in this thesis that our research task in future should be to formulate and systematize distributions of Chinese and English synonyms in a variety of differentiation standards by means of synonymous semantic fields.
\end{abstract}

KEYWORD: comparison; Chinese-English synonyms; comparability; differentiation standards; synonymous semantic field

\section{COMPARABILITY OF CHINESE-ENGLISH SYNONYMS}

From the general characteristics of the language, human natural language is a tool for people to express and communicate; it is a symbol system combined with pronunciation and meanings. Therefore, different languages can be compared [1]. However, language is a complex symbol system, and the comparison of a kind of language with another language as a whole is unrealistic [2]. A detailed comparison study is generally always based on the comparison of some parts between two language systems, but we must make sure that the two parts of the language phenomena that we select from the two language systems can be compared, and also should have made more sense.

Both Chinese language and English language have a symbol system of phonetic-semantic combination, thus they can be compared. The comparative study is based on the synonym level of the two kinds of vocabulary systems. Chinese and English synonyms are very rich; it is recognized by the theoretical circle. The study on the comparison between Chinese and English synonyms, no doubt, is of great significance, especially helping students to make correct choices in the teaching of English writing, English-Chinese translation. The purpose of our study is to, based on the academic research results, study Chinese and English synonyms and solve the word choice problems in Chinese-English translation and English writing.

It must be pointed out that our study of ChineseEnglish synonyms (phrase synonyms being outside the scope of this study; only including modern Chinese and modern English) is mainly limited to the semantic level, generally not considering synonyms with morphological and phonological levels. Synonyms here not only refer to the ones with completely the same meanings, but also include those synonyms with similar meanings, because synonyms with exactly the same meanings are few, their study ranges are narrow, and their study significance is relatively modest, especially in the choice of words.

\section{THE CONCEPT OF ENGLISH SYNONYMS}

As to the problem of the concept of synonyms, Chinese scholars generally believe that it refers to "the words with the same or similar meaning" in the language [3]. According to this definition, the scholars term synonyms with the same meaning (including the lexical meaning, grammatical meaning, color meaning) absolute synonyms or 
complete synonyms, and synonyms with basically the same meaning is called similar synonyms or relative synonyms. As the name suggests, absolute synonyms have the same connotative meaning in any context, and they can be interchangeable with each other, such as Chinese synonyms: yishang (clothes) - yifu, jidu (envy) - duji, shibing (soldiers) bingshi, huxiang (mutual) - xianghu, juanxincai (cabbage) - baoxincai. Chinese absolute synonyms have the small number, because every word has its existence value in language, for the sake of the needs of language expression. If two or more than two synonymous words have the complete meaning, then they will lose their reason to exist [4]. Similar synonyms refer to a similar meaning situation, mainly manifested in three aspects: on the grammatical meaning, they are the same; on the lexical meaning, they have the same or similar meaning; in a color sense, they are the same or different [3], such as: fuqing (father) - baba (daddy), gudong (promote) - sandong (incite), youliang (fine) - youxiu (excellent).

The traditional definition of English synonyms is "Synonyms are words different in sound and spelling, but most nearly alike or exactly the same in meaning". Webster's New Synonyms Dictionary (1978) gives a definition of synonym: "Synonym is one of the two or more words in the English language which have the same or very nearly the same essential fancy". Although the two definitions have caused many disputes, they have pointed out that synonyms are the same or similar in meaning. Thus, English synonyms can be divided into two kinds: absolute synonyms and relative synonyms. The so-called absolute synonyms refer to those that are exactly the same in meaning and usage and can be replaced in any context. English absolute synonyms appear more in formal and technical vocabulary, such as words fricative and spirant in phonetics, toluene and methylbenzene in chemistry [5]. Relative synonyms are also called loose synonyms, and their meanings are only partially overlapped and they have the same meaning only in some contexts, such as English words "big, large, and huge". As the most widely used language in the world, English has the largest number of relative synonyms in the world. Colorful English synonyms can be used to accurately reflect the subtle differences between objective things, effectively express people's world outlook and values, avoid repetition and monotonousness, and enrich expression means [6].

It is not hard to understand that both Chinese and English synonyms are basically the same in concept and classification, and the difference is not too big. To be convenient to express, whether absolute synonyms or relative synonyms or complete synonyms or similar synonyms, we call all of them synonyms in the following statement. Of course, we should realize that complete or absolute equivalents are impossible - so-called complete or absolute synonyms are conditionally the same synonyms. Needless to say, wise experts have paid attention to this point.

Some words have the same or similar meaning but are different in parts of speech. Can these types of words be incorporated into the synonym category? It is generally thought that synonyms not only have the same denotative meaning, but also the parts of speech must be the same. A verb and an adjective can't be synonyms, and an adverb can't be synonymous with a noun. For example, "end" and "final" cannot be synonyms, because the former is a verb but the latter is a noun. Throughout English synonyms dictionary such as Oxford Learner's Thesaurus: A Dictionary of Synonyms, Webster's New Dictionary of Synonyms, New Generic Collins Dictionary, and Concise English Synonym Thesaurus, we find that the synonyms listed in them are the same in grammatical meaning (e.g., parts of speech being the same or similar), but the words with different parts of speech are not basically listed (except for multi-category words). Most Chinese scholars feel the same way. For example, from the above mentioned Ge's point of view, different parts of speech (or different grammatical meanings) are excluded from the synonyms. Actually controversy about this point has lasted for a long time, and the vast majority of scholars agree with Ge's opinion. Probably we Chinese learn from foreign linguists' point of view - "making foreign things serve China", or perhaps foreigners learn from Chinese linguists' argument - "making Chinese things serve foreign countries". But it is advisable that when comparing the synonym meanings, we should take into consideration words with the same meaning but different parts of speech, especially in the teaching practice. For example, comparing two English words "cross" and "across", we might as well try to use them as a synonym cluster to examine: the former that means "go from one side to another" is a verb, while the latter is used as a preposition or adverb. In fact, a similar situation exists in Chinese: "kunku" (poor) and "jiku" (sufferings), "yongjiu" (permanent) and "yongyuan" (forever), "huran" (suddenly) and "turan" (sudden). Hence, we comparatively agree with such a view: the main foundation of determining whether words are synonyms or not should be the semantic characteristics, rather than the grammatical features [7].

Through the above comparison, it is found that there is practically no difference between Chinese and English synonyms in concept, classification, and definition. Of course we should be sober-minded, because complete or absolute synonyms are not possible. The so-called complete or absolute synonyms are conditionally the same synonyms. On the question whether word items with the different 
part of speech but the same or similar meaning can be incorporated into synonym category or not, our view is: in a certain sense, the term of different parts of speech into a synonym cluster to discriminate can be conducive to the discrimination of some easily mixed words in Chinese or English.

\section{DIFFERENTIATION STANDARD OF CHINESE-ENGLISH SYNONYMS}

The standard or measure that examines the similarities and differences of synonyms is called the synonym differentiation standard. The differentiation standard plays a reference role in Chinese-English synonym discrimination. Around this reference, we can accurately discriminate Chinese-English synonyms from different perspectives. People often use the differentiation standards for reference to make the synonym structure analysis and the semanteme analysis in lexical semantics, especially the latter. In fact, the differentiation standards we are discussing here are similar to the difference project system in word discrimination, but they also have different places, because our focus is on the basis of difference project system in word discrimination, comparing the discrimination standards of ChineseEnglish synonyms, in order to find out the similarities and differences between the two languages, to facilitate the possible establishment of system project of English synonymous semantic fields using differentiation standards as the core, and to solve the word choice problem in a synonym cluster in English writing and translation, but not for the compilation of Chinese-English Synonym Dictionary.

The comparison between Chinese and English synonym differentiation standards is made in the following aspects: vocabulary meaning, including semantic intensity, semantic territory, semantic emphasis, commonness \& collectiveness, action content, and implication etc.; color meaning, including language style, lexical origination, figure, times, dialects, nationality, foreignness, praise \& derogation, likes \& dislikes, euphemism, and seriousness etc.; collocation meaning; distribution of position \& environment; core words.

Hence, it is easy to find that Chinese and English synonym differentiation standards are almost the same. However, we should understand that some standards in Chinese are used much more than in English, and vice versa; some standards basically have consistent use frequencies in English-Chinese synonym differentiation. On the other hand, there may be several differentiation measures or standards for word discrimination in a synonymous semantic field. It is pointed out that there is little difference in macro distribution of differentiation standards between Chinese and English synonymous semantic fields but much, in micro distribution. This phenomenon is mainly determined by the number of synonymous semantic fields or word clusters under a certain standard.

Therefore, our research task in future is to format and systematize differentiating distributions of Chinese and English synonyms, especially English synonyms, in terms of synonymous semantic fields for lexical analysis. In most cases, in both English and Chinese, synonym differentiation standards in a certain synonymous semantic field can be considered from several aspects, which is up to several subtle differences in terms of word basic meaning, additional meaning (color meaning), collocative meaning and context meaning. Accordingly, synonym differentiation standards have diversity, and this diversity should become the source power of formatted and systematized Chinese-English synonym differentiation standards.

\section{COMPARISON OF DIFFERENT TYPES OF SYNONYM DIFFRENTIATION STANDARDS}

In terms of differentiation standards of different types of synonym aggregation, some standards are similar; some are not the same; and some present a kind of crisscrossed phenomenon. And different types of synonym differentiation standards are not only suitable for analysis of Chinese synonyms, also for the discrimination of English synonyms.

If synonym aggregation is discriminated in terms parts of speech, according to Zhang (2005), synonyms can be divided into noun synonyms, verb synonyms, adjective synonyms, etc. The main differences of noun synonyms lie in semantic features referring to people or things, as well as other differences such as semantic domain size, commonness and aggregation, sememe focus, etc. The main differences between verb synonyms are in characteristics referring to action or change, action content (including doer, recipient, way, direction, method, result and so on), object, sememe weight, etc.. The major differences of adjective synonyms are in semantic features related to nature or status, and there are often other differences: sememe focus, sememe weight, modified center word, etc.

As a result, word items in a synonymous cluster can be discriminated from several aspects, not necessarily confined to a certain standard. For example, both "anzhao" in Chinese (according to) and "yiju" (in accordance with) are prepositions, referring to "describing the basis of behavior" alike. But the former is commonly used in a variety of styles without semantic color, and its object can be legal and non-legal words. The latter is often used in a written style with solemn color, especially in national laws and regulations and in military and political messages, and its object is also related to 
legal or non-legal words [7]. The two Chinese words are analyzed respectively from prepositional object, semantic focus, stylistic color, formal color, and semantic scope.

\section{THE IMPORTANCE OF CHINESE-ENGLISH SYNONYM COMPARISON STUDY}

Both Chinese language and English language are very rich in synonyms. Study on the comparison between Chinese and English synonyms, no doubt, is of great significance.

First, study on the comparison between ChineseEnglish synonyms helps students to tell the differences of synonyms in teaching Chinese as a foreign language. In the second language teaching, it has been proved to be a good means to properly use native language or some familiar media language to compare with the target language, greatly making students take no detours in learning, especially at the beginning of learning.

Second, study on the comparison between Chinese-English synonyms helps students to make correct choices in the teaching of English writing, English-Chinese translation. Through the teaching practice, we have found that higher vocational college students are relatively weak in synonym discrimination and word choice in English writing or translation from Chinese into English. Besides their own poor language foundation, the more important reason is that students cannot have a good understanding or grasp of the standards or measures to distinguish the Chinese and English synonyms, correspondence or incorrespondence. However, using synonym standards to differentiate synonyms in a statement or a discourse can solve the problem for selection of synonyms, so as to improve the quality of writing or translation from Chinese into English.

Finally, study on the comparison between Chinese-English synonyms helps to establish a synonym recognition system based on the differentiation standards. The difference of macroscopic distribution between Chinese and English synonym standards is not very big, but the micro distribution is much bigger, mainly because of the number of the synonymous semantic field in a certain standard or word group. If the distribution of Chinese-English synonyms in various standards can be formatted and systematized through the establishment of minimum synonymous semantic fields, undoubtedly it will be conducive to the discrimination between synonyms and advantageous to the synonym system.

\section{REFERENCES}

[1] Xu, Yulong. 1992. Contrastive Linguistics. Shanghai: Shanghai Foreign Language Education Press.

[2] Sridhar, S.N. 1981. Contrastive Analysis, Error and Interlanguage: Three Phases of One Goal, in Fisiak ed.

[3] Ge, Benyi. 1985. Studies on Chinese Vocabulary Jinan: Shandong Education Press.

[4] Wang, Wenbin. 2001. English Vocabulary Semantics. Hangzhou: Zhejiang Education Press.

[5] Feng, Shimei. 2002. English Lexicology. Beijing: China Water Conservancy Press.

[6] Evelyn, H. 1995.Vocabulary, Semantics and Language Education. Cambridge: Cambridge University Press.

[7] Zhang, Zhiyi. 2005. Xinhua Synonym Dictionary. Beijing: Commercial Press. 